\title{
2021 in review
}

\author{
As the COVID-19 pandemic continues to progress through 2021, microbiology remains in the public eye. Here, we \\ reflect on the content that we published over the past year, from SARS-CoV-2 to all other areas of the field.
}

A s 2021 comes to an end, we would like to continue the tradition of reflecting back on the microbiology content that we published this year. First and foremost, however, we would like to thank our authors, reviewers and readers for their continued trust in Nature Microbiology as a journal that seeks to publish the most significant advances across the broad discipline of microbiology, irrespective of the size of individual research fields.

We live in unbelievably exciting but also challenging times when it comes to microbiology research. We are still in the midst of one of the biggest infectious disease challenges in recent history, yet the speed at which we have started to understand the basic biology and pathogenesis of SARS-CoV-2 and have begun to develop and deploy effective therapeutics for COVID-19 is unprecedented. Perhaps one good thing to have emerged from the pandemic is the spotlight on microbiology, both socially and politically, which has, consequently, galvanized interdisciplinary research collaborations.

Our microbiology coverage this year has included several papers on SARS-CoV-2, from nomenclature to understanding the pathogenesis process and finding effective treatments. The emergence of SARS-CoV-2 variants has been a global concern, and the Virus Evolution Working Group of the World Health Organization announced a naming scheme that enables clear communication about SARS-CoV-2 variants of interest and concern ${ }^{1}$. This is important as it helps to prevent countries from being stigmatized as the origins of variants. As for COVID-19 pathogenesis, respiratory failure has been associated with increased mortality, and one study showed that in the lungs, SARS-CoV-2 load, anti-SARS-CoV-2 antibody responses and the microbiota are predictive of poor outcomes in patients who are severely ill with COVID-19 (ref. ${ }^{2}$ ). With regards to therapeutics, one in vivo study in ferrets reported prophylactic and infection-limiting efficacy for a promising oral SARS-CoV-2 antiviral, molnupiravir ${ }^{3}$, which is currently in phase $2 / 3$ clinical trials (NCT04575597).

Keeping true to our aim of publishing research from all areas of the field, our coverage of virology was not restricted to SARS-CoV-2 and included a study showing that the shape of a virus can determine the probability of both virus attachment to host cells and membrane fusion ${ }^{4}$. The study showed that filamentous virions had a higher probability of attaching to host cells, even when partially neutralized by antibodies. In the (pre)clinical space, phage therapy has been used to resensitize Acinetobacter baumannii to antibiotics ${ }^{5}$; a promising therapeutic approach against a top priority drug-resistant opportunistic bacterial pathogen.

Many of the articles we publish do not have a clinical slant and instead explore the fundamental principles governing microbial physiology and report methods to study these issues. We published, for example, a Brief Communication on the development of Biofilm $\mathrm{Q}^{6}$, a method allowing quantitative imaging in biofilms. In terms of cell division, one study proposed a new two-track model for how cell septation progresses in bacteria ${ }^{7}$, and another study explored why some archaea carry two FtsZ proteins, finding that they have distinct functions in division ring assembly and constriction ${ }^{8}$. Another study solved the complete structure of the intact bacterial flagellar basal body ${ }^{9}$, showing how 173 protein molecules assemble into a multi-molecular complex that goes all the way from the cytoplasm, across the inner membrane, cell wall and outer membrane, to the outside of the cell. Further structural work illuminated the spectacular filament-forming nitrite oxidoreductase multi-protein complex ${ }^{10}$, the first structure for this enzyme in global nitrogen cycles.

The indispensable role microbes play in our environment was highlighted in several papers. A study on freshwater communities in small ponds ${ }^{11}$ showed that heavy rainfall (indicative of climate change) can impact communities ranging from microbes to zooplankton over time. Another study showed that marine cyanobacteria can produce more hydrocarbons than oil spills ${ }^{12}$. While this was previously shown in vitro, the authors demonstrate this in situ and uncover a potential cryptic hydrocarbon cycle that allows bacteria to consume these hydrocarbons.

Work on the symbiosis between hosts and their complex microbial communities, or microbiota, has continued to progress at great speed. A study in Dinoflagellates revealed that the establishment of symbiosis is dictated by local innate immune suppression to prevent expulsion and promote niche formation, contributing to our understanding of how endosymbiotic relationships are maintained ${ }^{13}$. Another study looking at the gut microbiome demonstrated that Bifidobacterium bifidum can augment the activity of immune checkpoint inhibitors ${ }^{14}$ and, consequently, reduce tumour burden in murine models. In a separate publication, Bifidobacterium species found in breast milk were shown to produce aromatic lactic acids ${ }^{15}$, which may impact immune function in early life. On the other hand, high fat dietinduced alterations to gut microbiota were found to reduce antibiotic efficacy ${ }^{16}$ in a mouse model. This was associated with a decrease in the tryptophan metabolite indole-3-acetic acid and a consequent inhibition of persister formation in vitro, highlighting the detrimental effects diet and the microbiota can have on our health. Beyond bacteria, archaeal gut communities have been analysed across 110 vertebrate species $^{17}$, revealing co-phylogeny, especially for mammalian herbivores. And beyond vertebrate hosts, a study characterizing the leaf microbiome (or phyllosphere) uncovered an essential plant gene for microbiota homeostasis ${ }^{18}$, encoding the plant NADPH oxidase RBOHD.

Bacterial pathogens cause significant morbidity and mortality worldwide, and treatment is often complicated by drug resistance. Tuberculosis, caused by mycobacteria, infected around 10 million people in 2020 (ref. ${ }^{19}$ ) and continues to be a grand challenge for human health worldwide. One study tracked the emergence and transmission of Mycobacterium abscessus ${ }^{20}$, a multidrug-resistant non-tuberculous mycobacterium that causes infections in people with cystic fibrosis. The authors identified the seven major circulating $M$. abscessus clones, reconstructed the spatiotemporal transmission networks and demonstrated a role for person-to-person transmission in M. abscessus epidemiology. Moreover, smokers and individuals without cystic fibrosis were revealed to be an important second human niche for M. abscessus. Another study was performed in vivo in mice with Mycobacterium 
canetti $^{21}$, a rare human pathogen, to investigate the origins of virulence in M. tuberculosis (Mtb). This led to the identification of mutations involved in $\mathrm{Mtb}$ persistence in humans. It is important to understand not only the pathogenesis of Mtb but also its response to antibacterial drugs. Using a network-based genetic screening approach, a study identified previously uncharacterized adaptations of Mtb to a first-line anti-tuberculosis drug ${ }^{22}$.

Parasites also continue to cause serious infections worldwide. A structural biology study in Plasmodium falciparum revealed the sequestration mechanism of placental malaria ${ }^{23}$, providing a path forward for potential interventions. Other studies analysed more fundamental principles of parasite molecular biology, showing that the spatial integration of transcription and mRNA splicing in a dedicated sub-nuclear compartment underpins monogenic antigen expression in African trypanosomes ${ }^{24}$ and that protein secretion by specialized secretory organelles (rhoptries) in Apicomplexa shares structural and genetic elements with the exocytic machinery of ciliates ${ }^{25}$.

In a previous Editorial, we argued that society must stop neglecting fungi ${ }^{26}$, as these microorganisms have a significant impact on human health, ecosystem function and the human microbiota, as well as many other areas. A Perspective outlined the nomenclature of fungal taxa known only from sequences ${ }^{27}$, which will aid communication across the scientific community and literature. We also published several studies on human fungal pathogens, including a paper showing that Mucorales fungi (which cause detrimental mucormycosis) produce a toxin that is similar to ricin and is required for fungal pathogenesis $^{28}$, and another revealing that vaginal epithelial cells have a biphasic response to Candida species, which consists of protective mitochondria-associated immune signalling and a species-specific damage-driven response ${ }^{29}$. Plant fungal pathogens pose a serious threat to agriculture and plant health, and a study in the corn smut fungus, Ustilago maydis, revealed that five effectors and two transmembrane proteins form a stable cell surface-exposed protein complex required for virulence ${ }^{30}$. Another study in the plant-pathogenic oomycete Phytophthora showed that it slices through the plant surface during host invasion using a mechanism that resembles the cutting of a sharp Japanese knife ${ }^{31}$.

In a year full of microbiological highlights, it was difficult to pick the ones to mention in this Editorial. We want to apologize to those authors whose work we did not mention but would like to reassure them that the selection was far from easy. We feel each and every paper that we published merits a mention (which, unfortunately, is not possible due to space constraints), but our main goal was not to provide a comprehensive list of papers but rather to highlight that we remain interested in all aspects of the field: from basic to translational microbiology; from bacteria to archaea, viruses, fungi and parasites; and from single cells to complex communities and their interactions with diverse hosts.

Research in microbiology has always been and will continue to be essential for humankind to understand, use and protect itself from the microbial world that dominates our planet. As we approach 2022, we would like to thank the scientific microbiology community once again for their hard and dedicated work in driving novel discoveries and advancing knowledge, and for their trust in our journal, in which we will continue to rigorously and fairly assess each manuscript that is submitted to us. We look forward to all of the upcoming exciting discoveries in microbiology and hope to share many of them with our readers next year and in the years to come.

Published online: 24 November 2021 https://doi.org/10.1038/s41564-021-01016-5
References

1. Konings, F. et al. Nat. Microbiol. https://doi.org/10.1038/s41564021-00932-w (2021).

2. Sulaiman, I. et al. Nat. Microbiol. https://doi.org/10.1038/s41564 021-00961-5 (2021)

3. Cox, R. M., Wolf, J. D. \& Plemper, R. K. Nat. Microbiol. https:// doi.org/10.1038/s41564-020-00835-2 (2021).

4. Li, T. et al. Nat. Microbiol. https://doi.org/10.1038/s41564-02100877-0 (2021).

5. Altamirano, F. G. et al. Nat. Microbiol. https://doi.org/10.1038/ s41564-020-00830-7 (2021)

6. Hartmann, R. et al. Nat. Microbiol. https://doi.org/10.1038/ s41564-020-00817-4 (2021).

7. Yang, X. et al. Nat. Microbiol. https://doi.org/10.1038/s41564020-00853-0 (2021)

8. Liao, Y., Ithurbide, S., Evenhuis, C., Löwe, J. \& Duggin, I. G. Nat. Microbiol. https://doi.org/10.1038/s41564-021-00894-z (2021).

9. Johnson, S. et al. Nat. Microbiol. https://doi.org/10.1038/s41564021-00895-y (2021).

10. Chicano, T. M. et al. Nat. Microbiol. https://doi.org/10.1038/ s41564-021-00934-8 (2021).

11. Shabarova, T. et al. Nat. Microbiol. https://doi.org/10.1038/ s41564-020-00852-1 (2021).

12. Love, C. R. et al. Nat. Microbiol. https://doi.org/10.1038/s41564020-00859-8 (2021).

13. Jacobovitz, M. R. et al. Nat. Microbiol. https://doi.org/10.1038/ s41564-021-00897-w (2021).

14. Lee, S.-H. et al. Nat. Microbiol. https://doi.org/10.1038/s41564 020-00831-6 (2021).

15. Laursen, M. F. et al. https://doi.org/10.1038/s41564-02100970-4 (2021).

16. Liu, Y. et al. Nat. Microbiol. https://doi.org/10.1038/s41564021-00912-0 (2021).

17. Youngblut, N. D. et al. Nat. Microbiol. https://doi.org/10.1038/ s41564-021-00980-2 (2021).

18. Pfeilmeier, S. et al. Nat. Microbiol. https://doi.org/10.1038/ s41564-021-00929-5 (2021)

19. Tuberculosis Fact sheet (WHO, 2021); https://www.who.int/ news-room/fact-sheets/detail/tuberculosis

20. Ruis, C. et al. Nat. Microbiol. https://doi.org/10.1038/s41564 021-00963-3 (2021)

21. Allen, A. C. et al. Nat. Microbiol. https://doi.org/10.1038/s41564021-00938-4 (2021).

22. Ma, S. et al. Nat. Microbiol. https://doi.org/10.1038/s41564-02000810-x (2021).

23. Ma, R. et al. Nat. Microbiol. https://doi.org/10.1038/s41564-02000858-9 (2021).

24. Faira, J. et al. Nat. Microbiol. https://doi.org/10.1038/s41564-02000833-4 (2021).

25. Aquilini, E. et al. Nat. Microbiol. https://doi.org/10.1038/s41564020-00854-z (2021).

26. Nat. Microbiol. https://doi.org/10.1038/nmicrobiol.2017.120 (2017).

27. Lücking, R. et al. Nat. Microbiol. https://doi.org/10.1038/s41564021-00888-x (2021).

28. Soliman, S. et al. Nat. Microbiol. https://doi.org/10.1038/s41564020-00837-0 (2021).

29. Pekmezovic, M. Nat. Microbiol. https://doi.org/10.1038/s41564021-00875-2 (2021).

30. Ludwig, N. et al. Nat. Microbiol. https://doi.org/10.1038/s41564021-00896-x (2021)

31. Bronkhorst, J. et al. Nat Microbiol. https://doi.org/10.1038/ s41564-021-00919-7 (2021). 